\title{
009 OP A SOCIOGRAM IS WORTH A THOUSAND WORDS: PROPOSING A METHOD FOR THE VISUAL ANALYSIS OF NARRATIVE DATA
}

D Contandriopoulos, ${ }^{1,2}$ C Larouche ${ }^{2,3 *}$. 'Université de Montréal, Faculté des sciences infirmières, Montréal, Québec, Canada; ${ }^{2}$ Institut de recherche en santé publique de I'Université de Montréal (IRSPUM), Montréal, Québec, Canada; ${ }^{3}$ Department of Anthropology, McGill University, Montréal, Québec, Canada *Presenting author.

10.1136/bmjopen-2015-UCLSymposiumAbstracts.9

This presentation proposes and showcases an innovative method for the visual analysis of narrative data. This method rests on three steps: the transformation of narrative data into relational data, the use of graph optimization algorithms derived from social network analysis (SNA), and, finally, the visual analysis of the resulting sociograms. This method was developed and pilot tested in the context of a research project about stakeholders' views on the strengths and problems of Quebec's health care system, and the solutions needed to increase its performance and sustainability.

SNA is a transdisciplinary methodological approach focused on understanding the structure of the relations that connect different elements. The scope of its application is very wide, from understanding the structure of molecular interactions and disease transmission in epidemiology to the analysis of kinship structures and community organization in anthropology. Although SNA has a long tradition in social sciences, its mainstream acceptance is recent.

This presentation begins with a brief summary of the specificity, origins and evolution of SNA tools, followed by a discussion on how the reliance on relational analysis differentiates SNA from other paradigms used in qualitative and quantitative analysis. It then provides an overview of the narrative data analysis method we developed and illustrates it via a case study on different perspectives of the Quebec health care system. Lastly, we outline the originality, potential and applicability of using SNA-based methods to analyse narrative data collected in qualitative health research.

We will argue here that examining how actors and their opinions constitute a network-like structure offers promising ways of interpreting data. In our research, the use of this method presented two main advantages. It provided powerful data visualization that facilitated the inductive identification of the underlying structure of our data. It also revealed the complexities of the links between differently positioned actors in the Quebec health care system that a personal attribute-based analytic method would have overlooked. 